\title{
ON REARRANGEMENT OF SERIES, II
}

P. H. DIANANDA (SINGAPORE)

1. Let $N_{1}, N_{2}, \ldots$ be a permutation of the positive integers. Then the series $a_{N_{1}}+a_{N_{2}}+\ldots$ is a rearrangement in the order of its terms of the series $a_{1}+a_{2}+\ldots$ (hereafter called series $\mathrm{A}$ ) of complex terms.

In a recent note [1] we generalized a theorem of Jasek [2] to

THEOREM 1. Suppose that

$$
n a_{n} \rightarrow 0
$$

$$
\text { as } \quad n \rightarrow \infty
$$

and $n a_{N_{n}} \rightarrow 0$ as $n \rightarrow \infty$. Then

$$
\left(a_{N_{1}}+\ldots+a_{N_{n}}\right)-\left(a_{1}+\ldots+a_{n}\right) \rightarrow 0 \quad \text { as } n \rightarrow \infty .
$$

Jasek's theorem follows as a special case if the series A is convergent. In [1], using Theorem 1, we obtained a solution of Jasek's problem P300 [2].

In this note we shall prove

THEOREM 2. For (2) to be true for every series A satisfying (1), a necessary and sufficient condition (NSC) is

$$
\sum_{r>n \geqslant N_{r}} \frac{1}{N_{r}}=O(1) \quad \text { as } \quad n \rightarrow \infty .
$$

Jasek's problem P 301 [2] may be restated as

Find an NSC that (2) be true for every convergent series A satisfying (1). Theorem 2, while not a solution of the above, solves a related problem. In what follows we shall, for brevity, write $\sum^{\prime}$ for $\sum_{r>n \geqslant N_{r}}$ and $\Sigma^{\prime \prime}$ for $\sum_{r \leqslant n<N_{r}}$.

2. To prove Theorem 2 , we note first that

$$
\left(a_{N_{1}}+\ldots+a_{N_{n}}\right)-\left(a_{1}+\ldots+a_{n}\right)=\sum^{\prime \prime} a_{N_{r}}-\sum^{\prime} a_{N_{r}} .
$$


From (1), it easily follows that

$$
\sum^{\prime \prime} a_{N_{r}} \rightarrow 0 \text { as } \quad n \rightarrow \infty
$$

Hence, from (4), we have

THEOREM 3. For (2) to be true for the series A satisfying (1), an NSC is

$$
\sum^{\prime} a_{N_{r}} \rightarrow 0 \text { as } \quad n \rightarrow \infty \text {. }
$$

Theorem 2 follows from Theorem 3 and the following lemmas: LEMMA 1. (1) and (3) $\Rightarrow(6)$.

LEMMA 2. Let (3) be false and $a_{n}=1 / n s_{n}$, where

$$
s_{p}=1+\max _{n \leqslant \nu} \sum \frac{1}{N_{r}} .
$$

Then (1) is true and (6) false.

The proof of Lemma 1 becomes obvious if we note that $N_{n} \rightarrow \infty$ with $n$. Hence

To prove Lemma 2, we note first that $s_{N_{r}} \leqslant s_{n}$ for $r>n \geqslant N_{r}$.

$$
\sum^{\prime} a_{N_{r}}=\sum^{\prime} \frac{1}{N_{r} s_{N_{r}}} \geqslant \sum^{\prime} \frac{1}{N_{r} s_{n}} .
$$

But $s_{n} \rightarrow \infty$ with $n$, since (3) is false. Hence (1) is true and

$$
\limsup _{n \rightarrow \infty} \sum^{\prime} a_{N_{r}} \geqslant \limsup _{n \rightarrow \infty} \sum^{\prime} \frac{1}{N_{r} s_{n}}=1 \text {. }
$$

Thus (6) is false. This concludes the proof.

Remark 1. Theorem 2 remains true if the word "series" is replaced by the phrase "divergent series". This follows since

$$
s_{n} \leqslant 1+\left(\frac{1}{1}+\ldots+\frac{1}{n}\right) \sim \log n
$$

so that the series $\mathrm{A}$, where $a_{n}=1 / n s_{n}$, is divergent.

Remark 2. In each of Theorems 1 and 3 condition (1) may be replaced by condition (5).

3. The following results are easily proved:

THEOREM 4. Condition (3) is an NSC for

$$
\left(a_{N_{1}}+\ldots+a_{N_{n}}\right)-\left(a_{1}+\ldots+a_{n}\right)=O(1) \quad \text { as } \quad n \rightarrow \infty
$$

to be true for every series A satisfying the condition

$$
n a_{n}=O(1) \quad \text { as } \quad n \rightarrow \infty .
$$

THEOREM 5. If

$$
\sum^{\prime \prime} \frac{1}{N_{r}} \rightarrow 0 \quad \text { as } \quad n \rightarrow \infty
$$

for (2) to be true for every series A satisfying (8), an NSC is

$$
\sum \frac{1}{N_{r}} \rightarrow 0 \quad \text { as } \quad n \rightarrow \infty \text {. }
$$

Note. The sums in (9) and (10) have equal numbers of terms. Consequently (10) implies (9). Thus (8) and (10) imply (2).

Remark 3. Theorems 4 and 5 are also true with "series" replaced by "divergent series".

The following related problems are of interest:

P 377. Find results corresponding to Theorems 4 and 5 for "convergent series".

P 378. (a) Find an NSO that (7) be true for every (i) series, (ii) divergent series, (iii) convergent series A satisfying (5) and (8). (b) Solve (a) with (7) replaced by (2). (c) Solve (a) with (5) and (8) replaced by (1).

Addendum (1 November 1961). An NSC that $a_{N_{1}}+a_{N_{2}}+\ldots$ converges for every convergent series $A$ has been found by Kronrod [3] I am indebted to B. Jasek for informing me of this.

Addendum (in proof). The same NSC has also been found by Levi [4]. I am indebted to U. C. Guha for drawing my attention to [4].

\section{REFERENCES}

[1] P. H. Diananda, On rearrangement of series, Proceedings of the Cambridge Philosophical Society 58 (1962), p. 158-159.

[2] B. Jasek, Über Umordnung von Reihen, Colloquium Mathematicum 7 (1960), p. $257-259$

[3] А. С. Кронрод, О перестановках иленьв ивисловых рядов, Математический Сборник 18 (60) (1946), p. 237.280.

[4] F. W. Levi, Rearrangement of convergent series, Duke Mathematical Journal 13 (1946), p. $579-585$.

\section{DEPARTMENT OF MATHEMATICS}

UNIVERSITY OF SINGAPORE

Rę̧u par la Rédaction le 28.9.1961 